\title{
The association of ideal cardiovascular health with incident type 2 diabetes mellitus: the Multi-Ethnic Study of Atherosclerosis
}

\author{
Joshua J. Joseph ${ }^{1}$ - Justin B. Echouffo-Tcheugui ${ }^{2,3} \cdot$ Mercedes R. Carnethon $^{4}$. \\ Alain G. Bertoni ${ }^{5} \cdot$ Christina M. Shay $^{6} \cdot$ Haitham M. Ahmed $^{7} \cdot$ Roger S. Blumenthal ${ }^{7}$. \\ Mary Cushman ${ }^{8} \cdot$ Sherita H. Golden ${ }^{1}$
}

Received: 8 March 2016 / Accepted: 6 May 2016/Published online: 8 June 2016

(C) Springer-Verlag Berlin Heidelberg 2016

\begin{abstract}
Aims/hypothesis Levels of ideal cardiovascular health (ICH) and incident type 2 diabetes mellitus have not been examined in a multiethnic population. We assessed the total and race/ethnicity-specific incidence of diabetes based on American Heart Association (AHA) ICH components.

Methods Incident diabetes was assessed among 5341 participants in the Multi-Ethnic Study of Atherosclerosis without prevalent diabetes between 2002 and 2012. ICH components (total cholesterol, BP, dietary intake, tobacco use, physical activity and BMI) were assessed at baseline and participants were categorised as having ideal, intermediate or poor cardiovascular health, as defined by the AHA 2020 impact goals. We developed a scoring system based on the number of ICH components ( $0-1$ 'poor', $2-3$ 'intermediate', and $\geq 4$ 'ideal'). HRs were calculated using Cox models.

Results During a median follow-up of 11.1 years, we identified 587 cases of incident diabetes. After multivariable adjustment, participants with $2-3$ and $\geq 4$ ICH com-
\end{abstract}

Electronic supplementary material The online version of this article (doi:10.1007/s00125-016-4003-7) contains peer-reviewed but unedited supplementary material, which is available to authorised users.

Sherita H. Golden

sahill@jhmi.edu

1 Division of Endocrinology, Diabetes and Metabolism, Johns Hopkins University School of Medicine, 1830 E. Monument St, Suite 333, Baltimore, MD 21287, USA

2 Division of Endocrinology, Diabetes and Hypertension, Brigham and Women's Hospital, Harvard Medical School, Boston, MA, USA

3 Rollins School of Public Health, Emory University, Atlanta, GA, USA ponents vs $0-1$ components had a $34 \%$ lower (HR 0.66 ; $95 \%$ CI $0.54,0.80$ ) and a $75 \%$ lower (HR $0.25 ; 95 \%$ CI $0.18,0.35)$ diabetes incidence, respectively. There were significant differences by race/ethnicity: African-American and Hispanic-American participants with $\geq 4$ ICH components had diabetes incidence rates per 1000 person-years of $5.6(95 \%$ CI $3.1,10.1)$ and $10.5(95 \%$ CI $6.7,16.4)$, respectively, compared with $2.2(95 \%$ CI $1.3,3.7)$ among non-Hispanic white Americans.

Conclusions/interpretation Meeting an increasing number of AHA 2020 impact goals for dietary intake, physical activity, smoking, BP, cholesterol and BMI was associated with a dosedependent lower risk of diabetes with significant variation by race/ethnicity.

Keywords Adiposity $\cdot$ Blood pressure $\cdot$ Cholesterol . Diabetes · Dietary intake $\cdot$ Glucose $\cdot$ Ideal cardiovascular health $\cdot$ Physical activity $\cdot$ Race/ethnicity $\cdot$ Smoking
4 Department of Preventive Medicine, Feinberg School of Medicine, Northwestern University, Chicago, IL, USA

5 Division of Public Health Sciences, Wake Forest University Health Sciences, Winston-Salem, NC, USA

6 Gillings School of Global Public Health, University of North Carolina, Chapel Hill, NC, USA

7 Ciccarone Center for the Prevention of Heart Disease, Johns Hopkins University School of Medicine, Baltimore, MD, USA

8 Department of Medicine, University of Vermont College of Medicine, Burlington, VT, USA 


$\begin{array}{ll}\text { Abbreviations } \\ \text { AA } & \text { African-American } \\ \text { AHA } & \text { American Heart Association } \\ \text { CA } & \text { Chinese-American } \\ \text { CVD } & \text { Cardiovascular disease } \\ \text { HA } & \text { Hispanic-American } \\ \text { ICH } & \text { Ideal cardiovascular health } \\ \text { MESA } & \text { Multi-Ethnic Study of Atherosclerosis } \\ \text { MET } & \text { Metabolic equivalent of task } \\ \text { NHW } & \text { Non-Hispanic white }\end{array}$

\section{Introduction}

In 2010, the American Heart Association (AHA) published 2020 impact goals for cardiovascular health promotion with the aim of improving cardiovascular health and reducing deaths from cardiovascular disease (CVD) and stroke by $20 \%$ by the year 2020 [1]. As part of this initiative, the AHA defined the concept of ideal cardiovascular health (ICH), identifying seven health factors or behaviours that have been associated with healthy ageing without cardiovascular and other chronic diseases. These include total cholesterol, $\mathrm{BP}$, fasting plasma glucose, dietary intake, tobacco use, physical activity and BMI (Table 1) [1]. Many cardiovascular risk factors also confer a high risk of incident type 2 diabetes mellitus, including physical inactivity, obesity, unhealthy dietary habits, and, to a lesser extent, elevated BP and dyslipidaemia [2]. CVD is the leading cause of morbidity and mortality among individuals with diabetes. Individuals with diabetes have a threefold increase in cardiovascular mortality compared with those without diabetes [3]. Given the shared risk factors and burden of both CVD and diabetes in the USA, it is important to determine whether the 2020 impact goals for ICH will impact diabetes incidence.

Adherence to the various components of ICH varies by ethnicity [4-6]. While one study among American Indians suggested that individuals who met a greater number of ICH goals had a reduced risk of incident diabetes [6], to our knowledge this hypothesis has not been assessed in a multiethnic population.

We tested the hypothesis that participants with higher levels of cardiovascular health are less likely to develop diabetes based on the AHA metric in four racial/ethnic groups in the Multi-Ethnic Study of Atherosclerosis (MESA). We additionally examined whether the association of individual or overall cardiovascular health components with incident diabetes varies by race/ethnicity.

\section{Methods}

MESA is a population-based sample of 6814 men and women from four ethnic groups: non-Hispanic whites (NHW; 38\%), African-Americans (AA; 28\%), Chinese-Americans (CA; $12 \%$ ) and Hispanic-Americans (HA; 22\%). Participants were aged 45-84 years at baseline; those who reported a medical history of heart attack, angina, coronary revascularisation, pacemaker or defibrillator implantation, valve replacement, heart failure or cerebrovascular disease were excluded. Details of sampling and recruitment have been published elsewhere [7]. The study was approved by the institutional review boards of the six participating institutions.

During the baseline examination (2000-2002), standardised questionnaires and calibrated devices were used to obtain participant data including: demographics, occupation, level of

Table 1 Total cardiovascular health score and definitions of cardiovascular health ${ }^{\mathrm{a}}$

\begin{tabular}{|c|c|c|c|}
\hline Goal $/$ metric & Poor health & Intermediate health & Ideal health \\
\hline Current smoking, months & Yes & Former $\leq 12$ & Never or quit $\geq 12$ \\
\hline Total cholesterol, mmol/l (mg/dl) & $\geq 6.21(\geq 240)$ & $5.18-6.18(200-239)$, or treated to goal & $<5.18(<200)$ \\
\hline $\mathrm{BP}, \mathrm{mmHg}$ & Systolic $\geq 140$ or diastolic $\geq 90$ & $\begin{array}{c}\text { Systolic } 120-139 \text { or diastolic } \\
80-89 \text {, or treated to goal }\end{array}$ & $<120 /<80$ \\
\hline BMI, $\mathrm{kg} / \mathrm{m}^{2}$ & $\geq 30$ & $25.0-29.9$ & $<25$ \\
\hline Physical activity, MET min/week ${ }^{\mathrm{b}}$ & $<3$ & $3-449$ & $\geq 450$ \\
\hline Healthy diet score, components ${ }^{\mathrm{c}}$ & $0-1$ & $2-3$ & $4-5$ \\
\hline Fasting plasma glucose, $\mathrm{mmol} / \mathrm{l}(\mathrm{mg} / \mathrm{dl})^{\mathrm{d}}$ & $\geq 7.0(\geq 126)$ & $5.6-6.9(100-125)$, or treated to goal & $<5.6(<100)$ \\
\hline Points for ICH score per metric & 0 & 0 & 1 \\
\hline Points for total cardiovascular health score per metric & 0 & 1 & 2 \\
\hline
\end{tabular}

${ }^{\text {a }}$ Adapted from the AHA's strategic planning task force and statistical committee 2020 guidelines ${ }^{1}$

${ }^{\mathrm{b}}$ Adapted for MESA: MET min/week

${ }^{\mathrm{c}}$ Adapted for MESA: fruits and vegetables, $\geq 1.081$ (4.5 cups)/day; fish, two or more $98 \mathrm{~g}$ (3.5 ounce) servings per week (non-fried); fibre-rich whole grains, three or more $28 \mathrm{~g}$ (1 ounce)-equivalent servings/day; sodium, $<1500 \mathrm{mg} /$ day; sugar-sweetened beverages, $\leq 1884 \mathrm{~kJ}$ (1008 g)/week

${ }^{\mathrm{d}}$ Fasting plasma glucose was not used in the main analysis but was considered in the sensitivity analyses. 
education, tobacco use, alcohol consumption, medical conditions and current prescription medication. Race/ethnicity was assessed by self-report and categorised as NHW, non-Hispanic Black (AA), Hispanic (HA) or Chinese (CA). Current occupational status was classified into ten categories: homemaker, employed (full time, part time, on leave due to ill health, or on leave due to non-health related issues), unemployed ( $<6$ or $>6$ months) and retired (not working, working or volunteering). Education was classified into one of the following five categories: less than high school, completed high school, some college/technical school certificate or associate degree, bachelor's degree, and graduate or professional school. Smoking history was obtained using a questionnaire developed from the third National Health and Nutrition Examination Survey (NHANES III), the National Health Interview Survey (NHIS) and the Atherosclerosis Risk in Communities Study (ARIC). Current alcohol use was assessed as a binary variable by asking participants whether they presently drank alcoholic beverages. Calibrated devices were used to measure participants' weight, waist circumference and height; BMI was calculated as weight in kilograms divided by the square of height in metres. Resting seated BP was measured three times using a Dinamap Pro 100, automated oscillometric sphygmomanometer (Critikon, Tampa, FL, USA); the last two measurements were averaged for analysis.

Physical activity The MESA Typical Week Physical Activity Survey, adapted from the Cross-Cultural Activity Participation Study, was used to assess physical activity [8]. We used the intentional exercise variable (sum of walking for exercise, sports/dancing, and conditioning in metabolic equivalent of task [MET] min/week) with the following categorisation: 'poor' $<3$ MET min/week, 'intermediate' 3-449 MET min/week, 'ideal' $\geq 450 \mathrm{MET} \mathrm{min} /$ week. Given that $3 \mathrm{METs}$ and $6 \mathrm{METs}$ are the lower limits for moderate and vigorous intensity physical activity, our MET min/week categorisation is consistent with the minutes per week measure in the AHA guidelines [1].

Dietary assessment Dietary intake was assessed using the MESA food frequency questionnaire, a self-administered modified-Block-style 120-item questionnaire adapted from the Insulin Resistance Atherosclerosis Study instrument [7]. The MESA questionnaire had some slight differences compared with the 2020 guidelines regarding units of servings, requiring modification of the metrics. Components of the modified ideal diet score are: fruits and vegetables, $\geq 4.5$ cups (equivalent to 1.08 1)/day; fish, two or more $98 \mathrm{~g}$ (3.5 ounce) servings per week (non-fried); fibre-rich whole grains, three or more 28 g (1 ounce)-equivalent servings/day; sodium, $<1500 \mathrm{mg} /$ day; sugar-sweetened beverages, $\leq 1884 \mathrm{~kJ}$ $(1008 \mathrm{~g}) /$ week. Participants were given 1 point per dietary component at goal for a total score ranging from 0 to 5 .
Participants were classified as ideal (4-5 of 5 metrics), intermediate (2-3 of 5 metrics) or poor ( $0-1$ of 5 metrics).

Laboratory assessment Fasting blood samples were drawn and processed using a standardised protocol and sent to central laboratories at the Collaborative Studies Clinical Laboratory at Fairview University of Minnesota Medical Center (Minneapolis, MN, USA) for measurement of glucose, cholesterol and estimated glomerular filtration rate [9]. Serum glucose was measured by rate reflectance spectrophotometry using thin-film adaptation of the glucose oxidase method on a Vitros analyser (Ortho Clinical Diagnostics, Rochester, NY, USA). Total cholesterol was measured using a cholesterol oxidase method (Roche Diagnostics, Indianapolis, IN, USA) on the Roche Cobas Fara centrifugal analyser. Serum creatinine was measured by rate reflectance spectrophotometry using thin-film adaptation of the creatinine amidinohydrolase method on the Vitros analyser (Ortho Clinical Diagnostics) and calibrated to Cleveland Clinic. Creatinine was used to estimate glomerular filtration rate based on the Chronic Kidney Disease Epidemiology Collaboration equation [10].

Cardiovascular health Cardiovascular health was assessed with seven baseline metrics: smoking status, diet, physical activity, BMI, serum cholesterol and BP, with the addition of fasting plasma glucose in the sensitivity analyses [6]. Each baseline metric was scored and categorised as poor, intermediate or ideal, as specified by AHA recommendations, with consideration of medication use (i.e. antihypertensive, lipid-lowering, glucose-lowering) where appropriate [1]. Points were allocated for each of the seven baseline metrics with scores of 0 (poor or intermediate) or 1 (ideal) for each health behaviour (diet, smoking, physical activity, BMI) and health factor (BP, blood sugar, total cholesterol) (Table 1). We summed the metrics into the following categories: poor (0-1), intermediate (2-3) and ideal $(\geq 4)$ levels of cardiovascular health. Second, we used a previously developed scoring system [4] in which each cardiovascular health metric was given a point score of 0,1 or 2 to represent poor, intermediate or ideal health (Table 1), respectively. A total cardiovascular health score (excluding glucose) ranging from 0 to 12 was calculated as the sum of the individual cardiovascular health component scores. This score was classified into three levels as inadequate $(0-4)$, average $(5-8)$ or optimal $(9-12)$ cardiovascular health.

Diabetes definition Among those without prevalent diabetes at baseline, individuals newly using glucose-lowering medication or having fasting glucose $\geq 7 \mathrm{mmol} / 1(126 \mathrm{mg} / \mathrm{dl})$ at one of the four subsequent examinations (the last follow-up visit occurring in 2010-2012) were considered to have incident diabetes [11]. We defined time of incident diabetes as the midpoint between the last examination without diabetes and the examination at which diabetes developed. 
We were unable to distinguish between type 1 diabetes and type 2 diabetes, but in this age range, incident type 1 diabetes is extremely uncommon, so we assumed a predominance of type 2 diabetes in our population.

Statistical analysis We excluded participants with diabetes at baseline $(n=883)$, missing diabetes status at their last follow-up $(n=57)$ or missing data on baseline covariates $(n=526)$. The 583 participants excluded due to missing diabetes and covariate status had a higher percentage of AA, higher BMI, higher smoking, lower education, less physical activity and a higher rate of incident diabetes ( $p<0.05$ for all comparisons) (electronic supplementary material [ESM] Table 1). Descriptive statistics were used to compare the baseline characteristics of all participants (non-stratified) and by race/ethnicity, using appropriate parametric or non-parametric tests for continuous variables and the $\chi^{2}$ test for categorical variables. Among the seven ICH components, we did not consider the blood glucose metric in the main analysis, as diabetes was the endpoint of interest. We used the other six components of the ICH score [6] to develop the two aforementioned scoring systems for ICH and total cardiovascular health. Last, we assessed the association between individual cardiovascular health components and incident diabetes by comparing participants with intermediate or ideal status with those with poor status (reference group) at baseline for each of the six factors. Unadjusted diabetes incidence rates for cardiovascular health scores were calculated using person-time analysis assuming a Poisson distribution. Participants were censored at the last attended follow-up examination. Incidence rate ratios were assessed using the logrank test. Cox proportional hazards modelling was used to estimate HRs associated with the aforementioned classifications. Covariates in adjusted analyses included baseline study site, age, sex, race/ethnicity, education, occupational status, alcohol use and estimated glomerular filtration rate. The baseline number of cardiovascular health components and cardiovascular health values categorised as poor, intermediate or ideal were evaluated to estimate the proportion of cases in the population that might be attributable to suboptimal levels of cardiovascular health (population-attributable risk). The population-attributable risk was calculated using the formula $\mathrm{p}(\mathrm{RR}-1) /(1+\mathrm{p}[\mathrm{RR}-1])$, where $\mathrm{p}$ is the prevalence of individuals not in the low-risk group and RR (risk ratio, HR) is the associated multivariable-adjusted relative risk of those individuals. Upper and lower 95\% CIs of the population-attributable risk were derived using this formula and the upper and lower $95 \%$ CI estimates of the multivariable-adjusted risk ratio [12]. Given that the association of cardiovascular health attainment with diabetes risk may differ by age, sex and race/ethnicity, we tested for interaction by these factors with each cardiovascular health measure by inserting an interaction term in the model and using the likelihood ratio test. Statistical significance was defined as two-sided $\alpha<0.05$. Analyses were performed using Stata 13.1 software (StataCorp, College Station, TX, USA).

\section{Results}

Characteristics of the cohort The baseline characteristics of participants stratified by race/ethnicity revealed that characteristics were different across race/ethnicity. HA and AA participants had significantly higher BMI, systolic BP and fasting glucose and lower levels of ICH components compared with NHW (Table 2).

Incidence of diabetes During a median follow-up of 11.1 years, 587 participants developed incident diabetes (incidence rate 11.1 per 1000 person-years). Incidence rates were highest among HA (15.3) and AA (12.3) (Table 2). Participants who developed diabetes had higher baseline BMI (30.8 vs $27.5 \mathrm{~kg} / \mathrm{m}^{2}$ ), systolic BP (130 vs $125 \mathrm{mmHg}$ ) and fasting plasma glucose (5.61 vs $4.88 \mathrm{mmol} / \mathrm{l},[101 \mathrm{vs} 88 \mathrm{mg} / \mathrm{dl}]$ ) (comparisons $p<0.0001$; ESM Table 2).

Diabetes incidence rates per 1000 person-years in participants with $0-1,2-3$ or $\geq 4$ ICH components were $18.9(95 \%$ CI 15.9, 22.4), 12.1 (95\% CI 11.0, 13.3) and 4.4 (95\% CI 3.4, 5.7), respectively, with a risk ratio of incident diabetes per category of $0.52(95 \%$ CI $0.46,0.60)$ (Table 3). Incident diabetes rates decreased for every additional ICH component achieved in the overall cohort and each racial/ethnic group (Table 3).

ICH and incident diabetes The unadjusted and adjusted HRs for incident diabetes associated with baseline ICH are presented in Table 4. After adjustment, the HR for 5-6 vs 0 individual ICH components was 0.17 (95\% CI 0.08, 0.38), and the HRs for intermediate or ideal categories compared with the poor cardiovascular health category were $0.66(95 \%$ CI $0.54,0.80)$ and 0.25 (95\% CI 0.18, 0.35), respectively. Among the individual cardiovascular health components, ideal vs poor status was associated with a decreased risk of incident diabetes for BP (HR 0.53; 95\% CI 0.42, 0.68) and BMI (HR 0.25; 95\% CI $0.19,0.33$ ) in adjusted analyses and for physical activity (HR $0.82 ; 95 \%$ CI $0.67,1.00$ ) in unadjusted analyses (Table 5). We found no consistent evidence of interactions between cardiovascular health components and age or sex (ESM Table 3). Sensitivity analyses conducted using the total cardiovascular health score (ESM Tables 4-6, ESM Figure 1), including participants with missing dietary data $(n=5817)$ (ESM Table 7), the addition of baseline glucose as a covariate (ESM Table 8), or the inclusion of glucose as a cardiovascular health component (seven components) (ESM Table 9) revealed similar findings and remained significant. Ideal $(<5.6 \mathrm{mmol} / 1[<100 \mathrm{mg} /$ $\mathrm{dl}])$ vs intermediate $(5.6-7.0 \mathrm{mmol} / 1[100-<126 \mathrm{mg} / \mathrm{dl}])$ baseline blood glucose categories were associated with an $87 \%$ reduction in diabetes risk (ESM Table 10).

Cardiovascular health and incident diabetes by race/ethnicity There were significant differences by race/ethnicity in the 
Table 2 Characteristics of participants, by race/ethnicity, in MESA 2000-2012

\begin{tabular}{|c|c|c|c|c|c|}
\hline Characteristic & $\begin{array}{l}\text { Overall } \\
n=5348\end{array}$ & $\begin{array}{l}\text { NHW } \\
n=2277\end{array}$ & $\begin{array}{l}\mathrm{CA} \\
n=676\end{array}$ & $\begin{array}{l}\text { AA } \\
n=1293\end{array}$ & $\begin{array}{l}\text { HA } \\
n=1102\end{array}$ \\
\hline Age & $61.9(10.3)$ & $62.4(10.3)$ & $61.8(10.3)$ & $62.0(10.2)$ & $60.7(10.5)$ \\
\hline Female, $\%{ }^{\mathrm{a}}$ & 53.5 & 52.6 & 52.2 & 56.8 & 52.4 \\
\hline Current smoking, $\%$ & 14.0 & 13.1 & 5.3 & 19.8 & 14.1 \\
\hline Ideal cardiovascular diet score, $0-5^{\mathrm{b}}$ & $1.5(0.9)$ & $1.6(0.9)$ & $1.7(0.8)$ & $1.4(1.0)$ & $1.3(0.9)$ \\
\hline Current alcohol use, $\%$ & 58.3 & 73.4 & 31.8 & 52.7 & 49.9 \\
\hline Education $\geq$ bachelor's degree, $\%$ & 37.8 & 51.3 & 39.9 & 36.6 & 11.2 \\
\hline Employed, \% full time & 39.4 & 40.5 & 37.3 & 39.4 & 38.5 \\
\hline Exercise, physical activity MET min/week & $1603(2410)$ & $1725(2348)$ & $1156(1549)$ & $1824(2992)$ & $1365(2138)$ \\
\hline BMI, $\mathrm{kg} / \mathrm{m}^{2}$ & $27.9(5.3)$ & $27.5(5.0)$ & $23.8(3.3)$ & $29.7(5.8)$ & $29.0(4.7)$ \\
\hline Systolic BP, mmHg & $125(21)$ & $123(20)$ & $124(22)$ & $131(21)$ & $126(22)$ \\
\hline Diastolic BP, mmHg & $72(10)$ & $70(10)$ & $72(10)$ & $75(10)$ & $72(10)$ \\
\hline eGFR CKD-EPI, $\mathrm{ml} \mathrm{min} \min ^{-1} 1.73 \mathrm{~m}^{-2}$ & $78(16)$ & $74(14)$ & $80(15)$ & $80(17)$ & $80(15)$ \\
\hline Total cholesterol, mmol/1 (mg/dl) & $5.1(0.9), 195(35)$ & $5.1(0.9), 196(35)$ & $5.0(0.8), 193(32)$ & $4.9(0.9), 190(36)$ & $5.2(0.9), 199(36)$ \\
\hline Fasting plasma glucose, mmol/1 (mg/dl) & $5.00(0.61), 89(10)$ & $4.88(0.56), 88(10)$ & $5.10(0.56), 91(10)$ & $5.00(0.61), 90(11)$ & $5.05(0.61), 91(11)$ \\
\hline Incident diabetes ${ }^{c}$ & $11.1(10.3,12.1)$ & $8.3(7.2,9.5)$ & $11.6(9.3,14.5)$ & $12.3(11.0,15.0)$ & $15.3(13.1,17.8)$ \\
\hline \multicolumn{6}{|l|}{ Baseline no. of ICH components } \\
\hline Poor $(0-1), \%$ & 14.2 & 12.9 & 5.8 & 18.4 & 16.9 \\
\hline Intermediate (2-3), \% & 62.8 & 61.1 & 55.6 & 66.1 & 66.8 \\
\hline Ideal (4-6), \% & 23.0 & 26.0 & 38.6 & 15.5 & 16.3 \\
\hline \multicolumn{6}{|c|}{$\begin{array}{l}\text { Baseline individual cardiovascular health components } \\
\text { BP }\end{array}$} \\
\hline Poor, $\%$ & 24.1 & 20.3 & 23.2 & 30.9 & 24.8 \\
\hline Intermediate, $\%$ & 38.6 & 38.1 & 32.8 & 44.9 & 35.8 \\
\hline Ideal, \% & 37.3 & 41.7 & 43.9 & 24.2 & 39.5 \\
\hline \multicolumn{6}{|l|}{ BMI } \\
\hline Poor, $\%$ & 28.5 & 25.7 & 4.1 & 40.9 & 34.8 \\
\hline Intermediate, $\%$ & 40.2 & 40.5 & 29.6 & 38.8 & 47.4 \\
\hline Ideal, $\%$ & 31.3 & 33.8 & 66.3 & 20.3 & 17.8 \\
\hline \multicolumn{6}{|l|}{ Total cholesterol } \\
\hline Poor, $\%$ & 9.6 & 10.0 & 7.5 & 7.8 & 12.3 \\
\hline Intermediate, $\%$ & 42.9 & 46.6 & 38.8 & 40.2 & 40.7 \\
\hline Ideal, $\%$ & 47.5 & 43.4 & 53.7 & 52.0 & 47.0 \\
\hline \multicolumn{6}{|l|}{ Smoking } \\
\hline Poor, $\%$ & 14.0 & 13.1 & 5.3 & 19.8 & 14.2 \\
\hline Intermediate, $\%$ & 1.1 & 1.5 & 0.9 & 0.8 & 1.0 \\
\hline Ideal, $\%$ & 84.9 & 85.4 & 93.8 & 79.4 & 84.9 \\
\hline \multicolumn{6}{|l|}{ Dietary intake } \\
\hline Poor, $\%$ & 50.3 & 47.8 & 38.3 & 53.7 & 58.6 \\
\hline Intermediate, $\%$ & 48.2 & 50.6 & 60.8 & 44.6 & 40.0 \\
\hline Ideal, $\%$ & 1.5 & 1.6 & 0.9 & 1.8 & 1.4 \\
\hline \multicolumn{6}{|l|}{ Physical activity } \\
\hline Poor, $\%$ & 21.8 & 16.3 & 25.4 & 22.4 & 30.0 \\
\hline Intermediate, $\%$ & 13.9 & 14.0 & 15.4 & 13.7 & 13.1 \\
\hline Ideal, \% & 64.3 & 69.7 & 59.2 & 64.0 & 56.9 \\
\hline
\end{tabular}

Values are mean (SD) or percentages unless otherwise indicated

${ }^{a}$ All comparisons $p<0.001$ except those that are not significant; $p$ values calculated using $\chi^{2}$ (categorical variables), ANOVA (continuous variables) and logrank tests (incident diabetes)

${ }^{\mathrm{b}}$ Adapted for MESA, with 1 point given for: fruits and vegetables, $\geq 1.081$ (4.5 cups)/day; fish, two or more $98 \mathrm{~g}$ ( 3.5 ounce) servings per week (nonfried); fibre-rich whole grains, three or more $28 \mathrm{~g}$ ( 1 ounce)-equivalent servings/day; sodium, $<1500 \mathrm{mg} / \mathrm{day}$; sugar-sweetened beverages, $\leq 1884 \mathrm{~kJ}$ $(1008 \mathrm{~g}) /$ week

${ }^{\mathrm{c}}$ Rate per 1000 person-years $(95 \% \mathrm{CI})$

eGFR CKD-EPI, estimated glomerular filtration rate based on the Chronic Kidney Disease Epidemiology Collaboration

association of baseline ICH components with risk of diabetes (all $p<0.01$; ESM Table 2). In Table 4, ideal vs poor cardiovascular health was associated with a greater reduction in diabetes risk in NHW and CA $(87 \%$ and $88 \%)$ vs AA and HA $(66 \%$ and $50 \%)$ (Fig. 1). Among the individual cardiovascular health components, ideal vs poor status was significant for: $\mathrm{BP}$ in NHW, CA and AA, with the greatest association of risk reduction in NHW (HR 0.36; 95\% CI 0.23, 0.55 ); and BMI in all ethnicities, with no significant difference among the race/ethnicities (Table 5). 
Table 3 Diabetes incidence rates $(95 \% \mathrm{CI})$ per 1000 person-years for baseline levels of $\mathrm{ICH}^{\mathrm{a}}$

\begin{tabular}{|c|c|c|c|c|c|}
\hline Baseline ICH level & Overall & NHW & $\mathrm{CA}$ & AA & HA \\
\hline \multicolumn{6}{|l|}{ No. of ICH components } \\
\hline 0 & $21.8(12.9,36.9)$ & $24.9(11.9,52.3)$ & $\mathrm{NA}^{\mathrm{b}}$ & $12.8(4.1,39.8)$ & $34.8(13.1,92.7)$ \\
\hline 1 & $18.6(15.5,22.2)$ & $17.8(13.3,23.8)$ & $35.8(19.8,64.7)$ & $16.4(11.6,23.2)$ & $19.1(13.3,27.3)$ \\
\hline 2 & $13.0(11.4,14.8)$ & $10.1(8.0,12.8)$ & $18.3(12.2,27.3)$ & $14.1(11.0,18.0)$ & $15.1(11.6,19.6)$ \\
\hline 3 & $11.2(9.7,12.9)$ & $7.9(6.1,10.2)$ & $12.4(8.7,17.8)$ & $13.2(9.9,17.5)$ & $15.6(11.8,20.7)$ \\
\hline 4 & $4.7(3.5,6.3)$ & $1.9(1.0,3.7)$ & $4.7(2.4,9.0)$ & $5.8(3.1,10.8)$ & $12.3(7.8,19.6)$ \\
\hline $5-6$ & $3.6(2.0,6.4)$ & $2.9(1.2,7.0)$ & $5.2(1.9,13.7)$ & $4.2(0.6 .29 .5)$ & $2.8(0.4,20.2)$ \\
\hline Rate ratio per 1 unit increase & $0.71(0.66,0.76)$ & $0.61(0.54,0.69)$ & $0.58(0.47,0.71)$ & $0.81(0.70,0.94)$ & $0.84(0.73,0.98)$ \\
\hline \multicolumn{6}{|c|}{ Cardiovascular health category, ICH components } \\
\hline Poor $(0-1)$ & $18.9(15.9,22.4)$ & $18.5(14.1,24.3)$ & $34.5(19.1,62.3)$ & $16.0(11.5,22.3)$ & $20.1(14.4,28.2)$ \\
\hline Intermediate $(2-3)$ & $12.1(11.0,13.3)$ & $9.0(7.5,10.7)$ & $14.5(11.1,18.9)$ & $13.7(11.4,16.5)$ & $15.3(12.7,18.5)$ \\
\hline Ideal $(\geq 4)$ & $4.4(3.4,5.7)$ & $2.2(1.3,3.7)$ & $4.8(2.8,8.3)$ & $5.6(3.1,10.1)$ & $10.5(6.7,16.4)$ \\
\hline Rate ratio per 1 unit increase & $0.52(0.46,0.60)$ & $0.39(0.31,0.49)$ & $0.36(0.24,0.53)$ & $0.67(0.52,0.88)$ & $0.73(0.56,0.96)$ \\
\hline
\end{tabular}

${ }^{a} 1$ point for attainment of $\mathrm{ICH}$ and 0 points for intermediate or poor cardiovascular health per category were summed to create a total score ranging from 0 to 6 and categorised into poor ( $0-1$ ICH components), intermediate (2-3 ICH components) and ideal ( $\geq 4$ ICH components)

${ }^{\mathrm{b}}$ Unable to define incident diabetes rate due to lack of participants meeting criteria for the category

NA, not applicable

Population-attributable diabetes risk We evaluated diabetes risk by baseline number of cardiovascular health components and categories at baseline, compared with all others not in that group (Table 6). The baseline number of ICH components $2,3,4$ or $\geq 5$ were associated with a $30 \%, 23 \%, 63 \%$ and $66 \%$ lower diabetes incidence, respectively. Individuals in the ICH category at baseline (23\%) had a $65 \%$ lower incidence of diabetes, and three in five new cases of diabetes appeared attributable to not being in the ICH group at baseline.

\section{Discussion}

In this large, contemporary, multiethnic cohort study, the presence of an increasing number of ICH components at baseline

Table 4 Diabetes incidence HRs $(95 \% \mathrm{CI})$ for baseline levels of ICH

\begin{tabular}{|c|c|c|c|c|c|c|}
\hline Baseline ICH level & Unadjusted & Multivariable-adjusted $^{\mathrm{a}}$ & $\mathrm{NHW}^{\mathrm{a}}$ & $\mathrm{CA}^{\mathrm{a}}$ & $\mathrm{AA}^{\mathrm{a}}$ & $\mathrm{HA}^{\mathrm{a}}$ \\
\hline \multicolumn{7}{|l|}{ No. of ICH components } \\
\hline 0 & 1 (referent) & 1 (referent) & 1 (referent) & 1 (referent) $^{\mathrm{b}}$ & 1 (referent) & 1 (referent) \\
\hline 1 & $0.84(0.48,1.46)$ & $0.82(0.47,1.43)$ & $0.78(0.35,1.73)$ & $\mathrm{NA}^{\mathrm{b}}$ & $1.26(0.38,4.12)$ & $0.50(0.17,1.41)$ \\
\hline 2 & $0.59(0.34,1.01)$ & $0.59(0.34,1.01)$ & $0.47(0.21,1.02)$ & $\mathrm{NA}^{\mathrm{b}}$ & $1.08(0.34,3.45)$ & $0.38(0.14,1.05)$ \\
\hline 3 & $0.51(0.30,0.88)$ & $0.51(0.30,0.88)$ & $0.36(0.17,0.80)$ & $N A^{b}$ & $1.00(0.31,3.21)$ & $0.39(0.14,1.10)$ \\
\hline 4 & $0.21(0.12,0.39)$ & $0.22(0.12,0.40)$ & $0.09(0.03,0.25)$ & $N A^{b}$ & $0.44(0.12,1.61)$ & $0.31(0.10,0.94)$ \\
\hline $5-6$ & $0.16(0.07,0.36)$ & $0.17(0.08,0.38)$ & $0.14(0.04,0.45)$ & $\mathrm{NA}^{\mathrm{b}}$ & $0.30(0.03,2.90)$ & $0.07(0.01,0.61)$ \\
\hline$p$ for trend ${ }^{c}$ & $<0.0001$ & $<0.0001$ & $<0.0001$ & & $<0.01$ & $<0.05$ \\
\hline \multicolumn{7}{|c|}{ Cardiovascular health category, ICH components } \\
\hline Poor $(0-1)$ & 1 (referent) & 1 (referent) & 1 (referent) & 1 (referent) & 1 (referent) & 1 (referent) \\
\hline Intermediate $(2-3)$ & $0.64(0.53,0.78)$ & $0.66(0.54,0.80)$ & $0.52(0.37,0.72)$ & $0.39(0.20,0.76)$ & $0.85(0.58,1.24)$ & $0.74(0.50,1.09)$ \\
\hline Ideal $(\geq 4)$ & $0.24(0.17,0.32)$ & $0.25(0.18,0.35)$ & $0.13(0.07,0.24)$ & $0.12(0.05,0.28)$ & $0.34(0.17,0.68)$ & $0.50(0.28,0.88)$ \\
\hline$p$ for trend ${ }^{\mathrm{c}}$ & $<0.0001$ & $<0.0001$ & $<0.0001$ & $<0.0001$ & $<0.01$ & $<0.05$ \\
\hline
\end{tabular}

${ }^{a}$ Adjusted for age, education, sex, study site, race/ethnicity, occupational status, alcohol use and estimated glomerular filtration rate; race-stratified analyses were not adjusted for race

${ }^{\mathrm{b}} \mathrm{CA}$ excluded from analysis due to sample size

${ }^{\mathrm{c}} p$ for trend calculated using the logrank test

NA, not applicable 
Table 5 Diabetes HRs $(95 \%$ CI) by individual categories of baseline cardiovascular health components

\begin{tabular}{|c|c|c|c|c|c|c|}
\hline $\begin{array}{l}\text { Baseline cardiovascular } \\
\text { health component }\end{array}$ & Overall & Overall adjusted $^{\mathrm{c}}$ & $\mathrm{NHW}^{\mathrm{c}}$ & $\mathrm{CA}^{\mathrm{c}}$ & $\mathrm{AA}^{\mathrm{c}}$ & $\mathrm{HA}^{\mathrm{c}}$ \\
\hline \multicolumn{7}{|l|}{$\mathrm{BP}^{\mathrm{a}}$} \\
\hline Poor & 1 (referent) ${ }^{\mathrm{d}}$ & 1 (referent) & 1 (referent) & 1 (referent) & 1 (referent) & 1 (referent) \\
\hline Intermediate & $0.96(0.79,1.16)$ & $0.94(0.77,1.13)$ & $0.73(0.52,1.03)$ & $1.19(0.68,2.09)$ & $0.87(0.61,1.23)$ & $1.26(0.84,1.88)$ \\
\hline Ideal & $0.46(0.37,0.58)$ & $0.53(0.42,0.68)$ & $0.36(0.23,0.55)$ & $0.51(0.26,1.00)$ & $0.54(0.33,0.89)$ & $0.88(0.55,1.41)$ \\
\hline \multicolumn{7}{|l|}{$\mathrm{BMI}^{\mathrm{a}}$} \\
\hline Poor & 1 (referent) & 1 (referent) & 1 (referent) & 1 (referent) & 1 (referent) & 1 (referent) \\
\hline Intermediate & $0.52(0.44,0.62)$ & $0.54(0.45,0.65)$ & $0.56(0.41,0.76)$ & $0.45(0.23,0.90)$ & $0.55(0.39,0.78)$ & $0.42(0.30,0.59)$ \\
\hline Ideal & $0.22(0.17,0.28)$ & $0.25(0.19,0.33)$ & $0.24(0.15,0.38)$ & $0.16(0.08,0.33)$ & $0.23(0.12,0.43)$ & $0.21(0.11,0.39)$ \\
\hline \multicolumn{7}{|l|}{ Total cholesterol $^{\mathrm{a}}$} \\
\hline Poor & 1 (referent) & 1 (referent) & 1 (referent) & 1 (referent) & 1 (referent) & 1 (referent) \\
\hline Intermediate & $1.04(0.79,1.39)$ & $1.00(0.75,1.33)$ & $0.76(0.49,1.18)$ & $1.19(0.50,2.83)$ & $1.19(0.63,2.26)$ & $1.06(0.61,1.85)$ \\
\hline Ideal & $0.90(0.68,1.19)$ & $0.87(0.65,1.16)$ & $0.72(0.46,1.15)$ & $0.67(0.28,1.61)$ & $0.84(0.44,1.61)$ & $1.20(0.69,2.08)$ \\
\hline \multicolumn{7}{|l|}{ Smoking $^{\mathrm{a}}$} \\
\hline Poor & 1 (referent) & 1 (referent) & 1 (referent) & 1 (referent) & 1 (referent) & 1 (referent) \\
\hline Intermediate & $1.30(0.63,2.69)$ & $1.13(0.55,2.34)$ & $0.66(0.20,2.18)$ & $\mathrm{NA}^{\mathrm{b}}$ & $1.64(0.38,7.05)$ & $1.92(0.57,6.46)$ \\
\hline Ideal & $0.97(0.77,1.22)$ & $0.99(0.78,1.26)$ & $0.82(0.55,1.23)$ & $0.76(0.31,1.86)$ & $1.11(0.71,1.74)$ & $1.00(0.62,1.62)$ \\
\hline \multicolumn{7}{|l|}{ Dietary intake $^{\mathrm{a}}$} \\
\hline Poor & 1 (referent) & 1 (referent) & 1 (referent) & 1 (referent) & 1 (referent) & 1 (referent) \\
\hline Intermediate & $0.98(0.83,1.16)$ & $1.15(0.97,1.37)$ & $1.13(0.83,1.54)$ & $1.21(0.74,1.96)$ & $1.10(0.79,1.53)$ & $1.19(0.86,1.66)$ \\
\hline Ideal & $1.23(0.67,2.25)$ & $1.70(0.92,3.12)$ & $1.49(0.46,4.80)$ & $0.64(0.08,4.96)$ & $2.55(1.03,6.47)$ & $1.17(0.28,4.89)$ \\
\hline \multicolumn{7}{|l|}{ Physical activity ${ }^{\mathrm{a}}$} \\
\hline Poor & 1 (referent) & 1 (referent) & 1 (referent) & 1 (referent) & 1 (referent) & 1 (referent) \\
\hline Intermediate & $0.95(0.73,1.24)$ & $1.08(0.83,1.41)$ & $1.30(0.83,2.03)$ & $1.48(0.72,3.06)$ & $1.11(0.63,1.93)$ & $0.71(0.40,1.25)$ \\
\hline Ideal & $0.82(0.67,1.00)$ & $0.98(0.80,1.19)$ & $0.83(0.57,1.21)$ & $1.23(0.70,2.16)$ & $1.26(0.84,1.90)$ & $0.95(0.66,1.36)$ \\
\hline
\end{tabular}

${ }^{\mathrm{a}}$ See Table 1 for description of poor, intermediate and ideal classifications of each individual cardiovascular health component

${ }^{\mathrm{b}} \mathrm{CA}$ excluded from analysis due to sample size

${ }^{\mathrm{c}}$ Adjusted for age, education, sex, study site, race/ethnicity, occupational status, alcohol use, estimated glomerular filtration rate and other cardiovascular health components; race-stratified analysis was not adjusted for race

${ }^{\mathrm{d}}$ HRs calculated using Cox proportional hazards modelling with the poor category as the referent group

NA, not applicable

was strongly and inversely associated with incident diabetes. The effect of ICH was graded: participants with intermediate or ideal vs poor cardiovascular health had a $34 \%$ and $75 \%$ lower diabetes incidence, respectively, which is consistent with previous studies $[12,13]$. In analyses stratified by race/ethnicity, the greatest reductions in diabetes risk based on ICH components were seen in NHW and CA. Overall, three out of five cases of diabetes in this middle-aged population appeared attributable to not having ICH at baseline. If these associations are causal, diabetes could be prevented by attainment of at least four ICH components. Compared with previous studies examining the combined effects of multiple risk factors on the incidence of diabetes [12, 13], we used a novel AHA concept that may be more adapted for translation of prevention policies that encompass both diabetes and CVD into practice. Public health interventions over the last 30 years have led to plateauing of diabetes incidence among NHW, but among AA and HA there remains a continued ascent in incident diabetes [14]. Overall, these findings support promotion of and adherence to the AHA 2020 impact goals to lower diabetes incidence among all races/ethnicities, but emphasise the importance of tailoring interventions to prevent diabetes in racial/ethnic minorities given the observed disparities.

ICH goals and incident diabetes Our study is the first multiethnic analysis to assess the association of baseline ICH with incident diabetes. A previous study among American Indians showed that participants who achieved $0-1 \mathrm{ICH}$ goals compared with those who achieved $2-3$ or $\geq 4$ ICH goals had a $60 \%$ and $89 \%$ lower odds of developing diabetes [6], compared with the $34 \%$ (2-3 ICH goals) and $75 \%$ ( $\geq 4$ ICH goals) lower diabetes incidence compared with $0-1$ ICH goals in our study. The observed difference is likely related to the inclusion of glucose among the ICH components in the Fretts et al study 


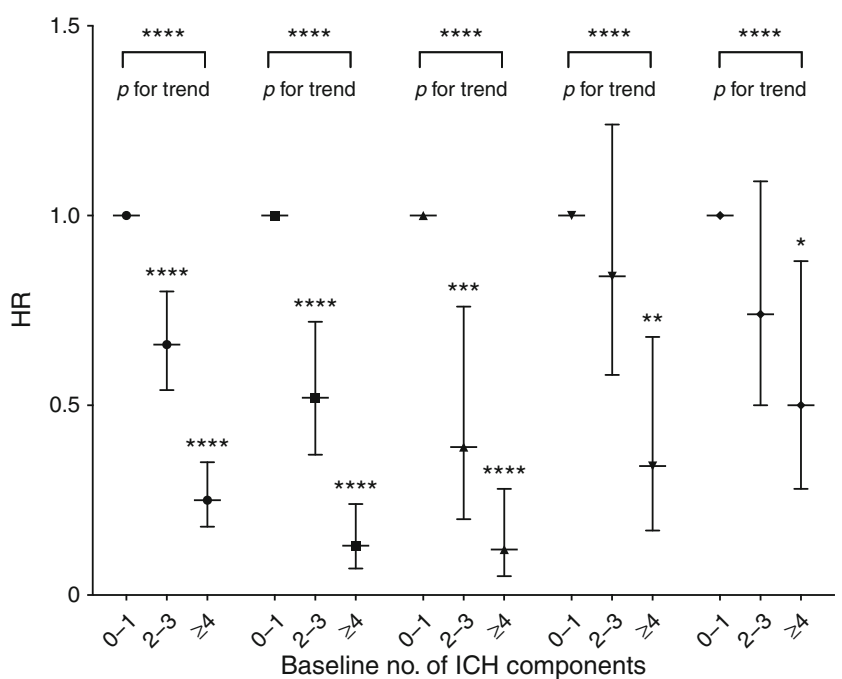

Fig. 1 Diabetes incidence HRs for ICH. ICH was classified as poor $(0-1$ ICH components) (referent group, $\mathrm{HR}=1$ ), intermediate (2-3 ICH components) and ideal ( $\geq 4 \mathrm{ICH}$ components) cardiovascular health. Circles, all races/ethnicities; squares, NHW participants; up-pointing triangles, CA participants; down-pointing triangles, AA participants; diamonds, HA participants. Cox modelling and logrank test ( $p$ for trend) with statistical significance for $* p<0.05,{ }^{* *} p<0.01, * * * p<0.001$ and $* * * * p<0.0001$

[6], whereas we excluded glucose in our main analysis, as it is directly in the causal pathway to diabetes. However, sensitivity analyses including glucose as a component of cardiovascular health revealed a similar order of risk reduction: $-61 \%$ and $-86 \%$ lower diabetes risk of participants in the intermediate or ideal category compared with those in the poor category. In the Cardiovascular Health Study [12], among older US adults (age $>65$ years) low-risk lifestyle groups defined by physical activity, dietary score, smoking, alcohol use, BMI and waist circumference were associated with an $89 \%$ reduction in diabetes risk with five low-risk lifestyle factors, similar to our $89 \%$ reduction in risk with $\geq 4 \mathrm{ICH}$ components. In the National Institutes of Health-American Association of Retired Persons (NIH-AARP) Diet and Health Study, Reis et al [13] examined the association of low-risk lifestyle factors including BMI, diet, smoking, moderate alcohol consumption and regular physical activity with incident diabetes (self-report). The ORs for incident diabetes over 11 years in those with all five low-risk lifestyle factors at baseline were 0.28 $(95 \%$ CI $0.23,0.34)$ and 0.16 (95\% CI $0.10,0.24)$ for men and women, respectively, suggesting sex differences. These findings were similar to our ICH category ( $\geq 4$ ICH components), and the test for effect modification by sex in our analysis was non-significant. We extended these previous findings by assessing a multiethnic population, as the Cardiovascular Health Study is $88.6 \%$ NHW and the NIHAARP Diet and Health Study is $94.3 \%$ NHW, thus limiting generalisability of the finding to other racial/ethnic groups.

Racial/ethnic differences Consistent with previous US population-based studies [15], AA and HA in the MESA cohort had lower levels of ICH at baseline. We observed significant variation of the association of higher ICH with lower incident diabetes among racial/ethnic groups. NHW and CA had greater magnitudes of risk reduction and better cardiovascular health compared with AA and HA $(p<0.01)$. The lower prevalence of ICH [15], combined with lower magnitude of diabetes reduction with ICH in AA and HA, provide a potential explanation and intervention target for the disparities in diabetes prevalence among these groups.

Table 6 Risk of incident diabetes mellitus and population-attributable risk according to number of baseline ICH components and categorised by level of cardiovascular health

\begin{tabular}{llllll}
\hline $\begin{array}{l}\text { Cardiovascular } \\
\text { health }\end{array}$ & $\begin{array}{l}\text { Participants, \% } \\
(n=5348)\end{array}$ & $\begin{array}{l}\mathrm{HR}^{\mathrm{a}} \\
(95 \% \mathrm{CI})\end{array}$ & $\begin{array}{l}\mathrm{HR}^{\mathrm{b}} \\
(95 \% \mathrm{CI})\end{array}$ & $\begin{array}{l}\text { Population-attributable } \\
\text { risk, \% }{ }^{\mathrm{a}}\end{array}$ & $\begin{array}{l}\text { Population-attributable } \\
\text { risk, \% } \\
(95 \% \mathrm{CI})\end{array}$ \\
\hline
\end{tabular}

No. of baseline ICH components

$\begin{array}{llll}2 & 32.0 & 0.70(0.56,0.87) & 23(9,35) \\ 3 & 30.7 & 0.77(0.64,0.92) & 17(6,28) \\ 4 & 17.7 & 0.37(0.27,0.50) & 58(45,69) \\ \geq 5 & 5.4 & 0.34(0.19,0.63) & 64(36,80)\end{array}$

Cardiovascular health category, ICH components
Intermediate (2-3) $\quad 62.8$
Ideal $(\geq 4)$
23.1
$0.66(0.54,0.80)$
$16(9,24)$
$0.35(0.27,0.46)$
$59(47,68)$

\footnotetext{
${ }^{a}$ Compared with participants meeting a lower number of baseline ICH components. The population-attributable risk is the percentage of new cases of diabetes in the population attributable to not adhering to a specified number of baseline ICH components. Analyses were adjusted for age, education, sex, study site, race/ethnicity, occupational status, alcohol use and estimated glomerular filtration rate

${ }^{\mathrm{b}}$ Compared with participants meeting criteria for lower cardiovascular health status. The population-attributable risk is the percentage of new cases of diabetes in the population attributable to not meeting criteria for a specified cardiovascular health category. Analyses were adjusted for age, education, sex, study site, race/ethnicity, occupational status, alcohol use and estimated glomerular filtration rate
} 
Individual AHA cardiovascular health goals and incident diabetes Impaired glucose tolerance, impaired fasting glucose and $\mathrm{HbA}_{1 \mathrm{c}}$ in the prediabetic range (5.7-6.4\% [38.8 $-46.4 \mathrm{mmol} / \mathrm{mol}])$ are associated with the greatest risk of diabetes, as they reflect derangements in the pathway from normal glucose tolerance to diabetes [16, 17]. Among risk factors not directly in the causal pathway, BMI was the predominant risk factor for diabetes, with a $48 \%$ and $77 \%$ lower diabetes risk for overweight BMI and normal BMI, respectively, vs obese BMI. This is consistent with previous studies showing increasing BMI as the primary risk factor increasing diabetes prevalence in the USA over the last 30 years [18]. In the Diabetes Prevention Program (DPP), sustained weight loss was the primary driver of reduced diabetes risk and cardiometabolic improvement [19]. In US populations, the imbalance between caloric intake and energy expenditure is the primary driver of increasing BMI and may have even greater importance in some racial/ethnic groups due to a reduction in baseline energy expenditure [20].

Among the other individual components, normal BP $(<120 /$ $<80 \mathrm{mmHg}$ ) was associated with a $47 \%$ diabetic risk reduction in the overall cohort vs elevated BP ( $>140 / 90 \mathrm{mmHg}$ ), with NHW showing the greatest reduction in diabetes risk compared with the other racial/ethnic groups. In a prior study, BP elevation was associated with incident diabetes in AA and NHW in age-adjusted analyses, but the association was non-significant among AA after adjustment for other diabetic risk factors [21]. This corresponded with an earlier study in which mean BP was significantly correlated with fasting plasma insulin and rate of glucose disposal in whites, but not in blacks [22]. Contrary to the prior literature, in our study, BP remained significant in all racial/ethnic groups except for HA. Elevated BP and diabetes can both be mediated by inflammation and decreased antioxidants, which may partly explain the association between BP and diabetes [23].

Physical activity is beneficial in reducing incident diabetes in the majority of NHW observational studies [24]. There is a scarcity of data on physical activity alone and incident diabetes in racial/ethnic minority groups in the USA. In observational studies, AA and HA have lower physical activity levels than NHW, independently of social class [25]. In a study of AA women, vigorous physical activity alone was associated with a dosedependent reduction in risk of incident diabetes [26]. Further studies to understand the impact of physical activity on diabetes risk are warranted in US racial/ethnic minority groups.

Smoking, dietary intake and total cholesterol were not individually associated with incident diabetes in our analysis. In large multiethnic meta-analyses, smoking increased the risk of diabetes, with a pooled adjusted relative risk of $1.4(95 \% \mathrm{CI}$ $1.3,1.6)$ vs non-smokers [27]. Data on causal inference between smoking and the development of diabetes are inconsistent: some studies show impairment in insulin sensitivity [28, 29] and glucose tolerance [29, 30], while other studies conclude that causal inference is not likely, after adjusting for confounders including age and BMI [31]. Further complicating the relationship are data from smoking cessation studies showing worsened glucose metabolism in those with diabetes in the first 1-3 years after smoking cessation [32], and an increased risk of incident diabetes in smokers without diabetes who quit, with subsequent improvement to similar risk of that of non-smokers over 12 years [33]. Smokers are also more likely to have unhealthy behaviours and low socioeconomic status, which contribute to the risk of diabetes [27].

While there was no association of dietary intake with incident diabetes, we are unable to draw definitive conclusions because only $1.5 \%$ of participants had baseline ideal dietary intake. Components of the AHA diet score including fruits and vegetables, fibre-rich whole grains, decreased sodium and sugar-sweetened beverage intake, and adherence to components of the Dietary Approaches to Stop Hypertension (DASH) and the Mediterranean diet have been associated with improvements in glucose metabolism and decreased incident diabetes [34-38]. A meta-analysis of ten large prospective studies found that dietary patterns similar to the AHA dietary recommendations were associated with a $66 \%$ reduction in diabetes [39]. Notably, the AHA diet score does not take into account other forms of dietary intake associated with decreases in diabetes and CVD risk including dairy consumption [40-42] and glycaemic index/load [43-45].

We found no significant overall association between total cholesterol and incident diabetes in our analysis. Components of the lipid profile including triacylglycerols, HDL and non-esterified fatty acids, have been associated with incident diabetes [46].

Among the AHA cardiovascular health components, BMI is well studied in US racial/ethnic minorities, in whom weight loss has been shown to reduce diabetes risk [19]. The association of individual components including BP, physical activity, smoking, total cholesterol and dietary intake with diabetes has previously revealed inconsistent findings and would benefit from further study among US racial/ethnic minorities.

Strengths and limitations Strengths of our analysis include a moderately large, socioeconomically diverse, multiethnic US population with over a decade of follow-up, allowing broad generalisability of our findings. We used validated questionnaires and documentation of diabetes over time with fasting glucose, medication use and self-reported physician diagnosis. Nevertheless, there are several potential limitations. Physical activity and diet were self-reported; thus, misclassification and residual confounding by these variables may have occurred. The time frame queried was the last month prior to the baseline visit; so, depending on the season of examination, the prior month may not be representative of customary physical activity and dietary intake. Sample sizes varied for the racial/ ethnic groups, with power implications for detecting significant racial/ethnic interactions in stratified analyses, but the 
interaction terms for categories of cardiovascular health were significant $(p<0.01)$. As previously mentioned, we were unable to distinguish between type 1 diabetes and type 2 diabetes, so we assumed a predominance of type 2 incident diabetes in our population.

Conclusions Our study showed that increasing levels of ideal and overall cardiovascular health within the guidelines set forth by the AHA 2020 impact goals may reduce the burden of diabetes in the USA. Unfortunately, less than one in four participants in our overall cohort and less than one in six racial/ethnic minorities attained $\geq 4$ ICH components, which is similar to findings in other studies $[4,5,15]$. Given the racial/ethnic differences in attainment of $\mathrm{ICH}$, the lower magnitude of risk reduction with ICH and the increased burden of diabetes in racial/ethnic minorities, further research on promotion, attainment and ethnic differences of ICH in US racial/ ethnic minority groups is of paramount importance to lower the risk of CVD and diabetes.

Acknowledgements The authors thank the other investigators, staff and participants of the MESA study for their valuable contributions. A full list of participating MESA investigators and institutions can be found at www.mesa-nhlbi.org. We thank C. Bennet (Department of Medicine, Johns Hopkins University, Baltimore, MD, USA) for assistance with manuscript preparation.

Funding This research was supported by contracts N01-HC-95159 through N01-HC-95165 and N01-HC-95169 from the National Heart, Lung, and Blood Institute. JJJ was supported by an institutional training grant from the National Institute of Diabetes, Digestive, and Kidney Diseases (T32 DK062707). The funding sources had no role in the study design; in the collection, analysis, and interpretation of data; in the writing of the report; or in the decision to submit the paper for publication.

Duality of interest The authors declare that there is no duality of interest associated with this manuscript.

Contribution statement All authors fulfil the contribution requirements for authorship credit, including, for each author listed: substantial contributions to conception and design, acquisition of data, or analysis and interpretation of data; drafting the article or revising it critically for important intellectual content; and final approval of the version to be published. SHG is the guarantor of this work.

\section{References}

1. Lloyd-Jones DM, Hong Y, Labarthe D et al (2010) Defining and setting national goals for cardiovascular health promotion and disease reduction: the American Heart Association's strategic impact goal through 2020 and beyond. Circulation 121:586-613

2. Gress TW, Nieto FJ, Shahar E et al (2000) Hypertension and antihypertensive therapy as risk factors for type 2 diabetes mellitus. N Engl J Med 342:905-912

3. Taylor KS, Heneghan CJ, Farmer AJ et al (2013) All-cause and cardiovascular mortality in middle-aged people with type 2 diabetes compared with people without diabetes in a large U.K. primary care database. Diabetes Care 36:2366-2371

4. Kulshreshtha A, Vaccarino V, Judd SE et al (2013) Life's simple 7 and risk of incident stroke: the reasons for geographic and racial differences in stroke study. Stroke 44:1909-1914

5. Folsom AR, Yatsuya H, Nettleton JA et al (2011) Community prevalence of ideal cardiovascular health, by the American Heart Association definition, and relationship with cardiovascular disease incidence. J Am Coll Cardiol 57:1690-1696

6. Fretts AM, Howard BV, McKnight B et al (2014) Life's simple 7 and incidence of diabetes among American Indians: the Strong Heart Family Study. Diabetes Care 37:2240-2245

7. Bild DE (2002) Multi-Ethnic Study of Atherosclerosis: objectives and design. Am J Epidemiol 156:871-881

8. Bertoni AG, Whitt-Glover MC, Chung H et al (2008) The association between physical activity and subclinical atherosclerosis: the Multi-Ethnic Study of Atherosclerosis. Am J Epidemiol 169:444 454

9. Cushman M, Cornell ES, Howard PR et al (1995) Laboratory methods and quality assurance in the Cardiovascular Health Study. Clin Chem 41:264-270

10. Levey AS, Stevens LA, Schmid CH et al (2009) A new equation to estimate glomerular filtration rate. Ann Intern Med 150:604-612

11. American Diabetes Association (2010) Diagnosis and classification of diabetes mellitus. Diabetes Care 33(Suppl 1):S62-S69

12. Mozaffarian D, Kamineni A, Carnethon M et al (2009) Lifestyle risk factors and new-onset diabetes mellitus in older adults: the Cardiovascular Health Study. Arch Intern Med 169:798-807

13. Reis JP, Loria CM, Sorlie PD et al (2011) Lifestyle factors and risk for new-onset diabetes: a population-based cohort study. Ann Intern Med 155:292-299

14. Geiss LS, Wang J, Cheng YJ et al (2014) Prevalence and incidence trends for diagnosed diabetes among adults aged 20 to 79 years, United States, 1980-2012. JAMA 312:1218

15. Shay CM, Ning H, Allen NB et al (2012) Status of cardiovascular health in US adults: prevalence estimates from the National Health and Nutrition Examination Surveys (NHANES) 2003-2008. Circulation 125:45-56

16. Morris DH, Khunti K, Achana F et al (2013) Progression rates from $\mathrm{HbA}_{1 \mathrm{c}} 6.0-6.4 \%$ and other prediabetes definitions to type 2 diabetes: a meta-analysis. Diabetologia 56:1489-1493

17. Nathan DM, Davidson MB, DeFronzo RA et al (2007) Impaired fasting glucose and impaired glucose tolerance: implications for care. Diabetes Care 30:753-759

18. Menke A, Rust KF, Fradkin J et al (2014) Associations between trends in race/ethnicity, aging, and body mass index with diabetes prevalence in the United States: a series of cross-sectional studies. Ann Intern Med 161:328

19. Delahanty LM, Pan Q, Jablonski KA et al (2014) Effects of weight loss, weight cycling, and weight loss maintenance on diabetes incidence and change in cardiometabolic traits in the Diabetes Prevention Program. Diabetes Care 37:2738-2745

20. Gannon B, DiPietro L, Poehlman ET (2000) Do African Americans have lower energy expenditure than Caucasians? J Int Assoc Study Obes 24:4-13

21. Wei GS, Coady SA, Goff DC et al (2011) Blood pressure and the risk of developing diabetes in African Americans and whites: ARIC, CARDIA, and the Framingham Heart Study. Diabetes Care 34:873-879

22. Saad MF, Lillioja S, Nyomba BL et al (1991) Racial differences in the relation between blood pressure and insulin resistance. N Engl J Med 324:733-739

23. Das UN (2008) Risk of type 2 diabetes mellitus in those with hypertension. Eur Heart J 29:952-953 
24. Jeon CY, Lokken RP, Hu FB, van Dam RM (2007) Physical activity of moderate intensity and risk of type 2 diabetes: a systematic review. Diabetes Care 30:744-752

25. Crespo CJ, Smit E, Andersen RE et al (2000) Race/ethnicity, social class and their relation to physical inactivity during leisure time: results from the Third National Health and Nutrition Examination Survey, 1988-1994. Am J Prev Med 18:46-53

26. Krishnan S, Rosenberg L, Palmer JR (2008) Physical activity and television watching in relation to risk of type 2 diabetes: the Black Women's Health Study. Am J Epidemiol 169:428-434

27. Willi C, Bodenmann P, Ghali WA et al (2007) Active smoking and the risk of type 2 diabetes: a systematic review and meta-analysis. JAMA 298:2654-2664

28. Frati AC, Iniestra F, Ariza CR (1996) Acute effect of cigarette smoking on glucose tolerance and other cardiovascular risk factors. Diabetes Care 19:112-118

29. Piatti P, Setola E, Galluccio E et al (2014) Smoking is associated with impaired glucose regulation and a decrease in insulin sensitivity and the disposition index in first-degree relatives of type 2 diabetes subjects independently of the presence of metabolic syndrome. Acta Diabetol 51:793-799

30. Janzon L, Berntorp K, Hanson M et al (1983) Glucose tolerance and smoking: a population study of oral and intravenous glucose tolerance tests in middle-aged men. Diabetologia 25:86-88

31. Wareham NJ, Ness EM, Byrne CD et al (1996) Cigarette smoking is not associated with hyperinsulinemia: evidence against a causal relationship between smoking and insulin resistance. Metabolism 45:1551-1556

32. Lycett D, Nichols L, Ryan R et al (2015) The association between smoking cessation and glycaemic control in patients with type 2 diabetes: a THIN database cohort study. Lancet Diabetes Endocrinol 3:423-430

33. Yeh H-C, Duncan BB, Schmidt MI et al (2010) Smoking, smoking cessation, and risk for type 2 diabetes mellitus: a cohort study. Ann Intern Med 152:10-17

34. Liese AD, Nichols M, Sun X et al (2009) Adherence to the DASH diet is inversely associated with incidence of type 2 diabetes: the Insulin Resistance Atherosclerosis Study. Diabetes Care 32:1434-1436

35. Shirani F, Salehi-Abargouei A, Azadbakht L (2013) Effects of Dietary Approaches to Stop Hypertension (DASH) diet on some risk for developing type 2 diabetes: a systematic review and metaanalysis on controlled clinical trials. Nutrition 29:939-947
36. Martinez-Gonzalez MA, de la Fuente-Arrillaga C, Nunez-Cordoba JM et al (2008) Adherence to Mediterranean diet and risk of developing diabetes: prospective cohort study. BMJ 336:1348-1351

37. Salas-Salvado J, Bullo M, Babio N et al (2011) Reduction in the incidence of type 2 diabetes with the Mediterranean diet: results of the PREDIMED-Reus nutrition intervention randomized trial. Diabetes Care 34:14-19

38. Schwingshack1 L, Missbach B, König J, Hoffmann G (2015) Adherence to a Mediterranean diet and risk of diabetes: a systematic review and meta-analysis. Public Health Nutr 18:1292-1299

39. Esposito K, Kastorini C-M, Panagiotakos DB, Giugliano D (2010) Prevention of type 2 diabetes by dietary patterns: a systematic review of prospective studies and meta-analysis. Metab Syndr Relat Disord 8:471-476

40. Soedamah-Muthu SS, Ding EL, Al-Delaimy WK et al (2011) Milk and dairy consumption and incidence of cardiovascular diseases and all-cause mortality: dose-response meta-analysis of prospective cohort studies. Am J Clin Nutr 93:158-171

41. O'Connor LM, Lentjes MAH, Luben RN et al (2014) Dietary dairy product intake and incident type 2 diabetes: a prospective study using dietary data from a 7-day food diary. Diabetologia 57:909917

42. Yakoob MY, Shi P, Willett WC et al (2016) Circulating biomarkers of dairy fat and risk of incident diabetes mellitus among men and women in the United States in two large prospective cohorts. Circulation 133:1645-1654

43. Villegas R, Liu S, Gao Y-T et al (2007) Prospective study of dietary carbohydrates, glycemic index, glycemic load, and incidence of type 2 diabetes mellitus in middle-aged Chinese women. Arch Intern Med 167:2310-2316

44. Sluijs I, van der Schouw YT, van der A DL et al (2010) Carbohydrate quantity and quality and risk of type 2 diabetes in the European Prospective Investigation into Cancer and NutritionNetherlands (EPIC-NL) study. Am J Clin Nutr 92:905-911

45. Burger KNJ, Beulens JWJ, Boer JMA et al (2011) Dietary glycemic load and glycemic index and risk of coronary heart disease and stroke in Dutch men and women: the EPIC-MORGEN study. PLoS ONE 6, e25955

46. Pankow JS, Duncan BB, Schmidt MI et al (2004) Fasting plasma free fatty acids and risk of type 2 diabetes: the Atherosclerosis Risk in Communities Study. Diabetes Care 27:77-82 